\section{HEPATIC INVOLVEMENT OF TOXOCARIASIS IN CHILDREN}

\author{
L. Mihai ${ }^{1}$, C.M. Mihai ${ }^{1}$, R. Stoicescu ${ }^{2}$ \\ ${ }^{1}$ Pediatric, ${ }^{2}$ Microbiology and Parazitology, Ovidius \\ University, Constanta, Romania
}

Introduction: Toxocariasis is a helmintozoonosis caused by the infection of a human host by the larva of Toxocara canis and Toxocara catis, which are common nematodes in dogs and cats. The disease shows variable clinical manifestations including hepatomegaly, fever, anorexia, cough, nausea, vomiting and right upper quadrant pain. Hepatic involvement is common due to portal venous drainage of visceral organs.

Aim: To evaluate hepatic involvement in children infected with Toxocara canis, by assessing clinical, biochemical and imagistic findings.

Methods: The record cards of 9 children, 12 months to 12 years and 6 months of age (mean 5,8 years), with serological diagnosis of Toxocara canis infection were evaluated.

Results: The patients were divided in two groups: the patients presented risk factors $(\mathrm{N}=6)$, and the patients without risk factors $(\mathrm{N}=3)$. The hepatic involvement was proven clinically (hepatomegaly in 5 cases), biochemically (hepatic cytolisis in 6 cases) and sonographically (in 5 cases). Abdominal ultrasound revealed multiple hypoechoic areas in the livers of 5 patients. Hepatohilar lymphnode enlargement was present in 3 patients, 2 of whom also showed peripancreatic lymphnode enlargement. Hepatomegaly was present in 5 patients and splenomegaly in 2. Eosinophilia level range between normal values (2 cases) and high values (2 cases). There is not significant differences between the two groups regarding the clinical, biochemical and echographic hepatic manifestations.

Conclusion: The study confirms the varied hepatic involvement of toxocariasis in children. The presence of risk factors didn't have a determinant influence in clinical, biological and imagistic picture in studied patients.

\section{MEASUREMENT OF CRP, PCT, ESR AND WBC IN LOWER RESPIRATORY TRACT INFECTION}

R. Bejiqi ${ }^{1}$, R. Retkoceri' ${ }^{1}$, H. Bejiqi', M. Kelmendi', N. Zeka' ${ }^{1}$, L. Kryeziu' ${ }^{2}$, S. Borovci ${ }^{1}$

${ }^{1}$ Pediatrics Clinic, Intensive care Unit, ${ }^{2}$ Intensive Care, Univeristy Clinical Centre of Kosova, Prishtina, Albania

Background Acute lower respiratory tract infections are the most frequent reason for seeking medical attention in primary and secondary care. They are caused by viruses, bacteria or combination of both, present with similar symptoms. Evaluation of C-reactive protein (CRP), Procalcitonin (PCT), erythrocyte sedimentation rate (ESR) and white blood cells (WBC) and their relations are the best and fastest methods to decide needing for antimicrobial therapy. Although, they are golden rules in assessment of effective used antibiotics.

Methods Using preferred procedure we measured serum level of CRP, PCT, ESR, WBC in all children, admitted in intensive care unit with clinical signs of lover respiratory tract infection, in admission and after 5 and 10 days of treatment.

Results During the period 03.01.2008 to 03.07.2009. in PICU in Prishtina, Kosova, were treated 128 children, less than 6 months of age, with lower respiratorytractinfection, clinicallywith predomination of bronchiolitis (96 cases). In all children were done lab analyses; special point in differential diagnosis we used serum level of CRP, PCT, ERS and WBC in admission and after 5 days of hospitalization. As referential values are used international levels. All children with bronchopneumonia in admission and after 5 days have positive results in all tests but after 10 days of treatment the positive are only ESR and particularly WBC. Only a few children with bronchiolitis have positive all tests in admission and after 5 days (probably bronchiolitis complicated with bronchopneumonia), and they have been treated by antimicrobiotic. 